\title{
Appellationen aus Reichsitalien Ein Konfliktfeld zwischen Reichshofrat und Plenipotenz
}

Im ersten (und einzigen) Band seiner „Beyträge zum Reichsstaatsrechte Welscher Nation" führt der Göttinger Privatdozent Johann Anton Ludwig Seidensticker 1795 zum Thema der Reichsgerichtsbarkeit in Italien unter anderem aus, er werde "ausführlich $\mathrm{zu}$ beweisen suchen, wie unrichtig man der Plenipotenz eine eigene Gerichtsbarkeit beylege“. Damit wendet er sich ausdrücklich gegen die Darstellungen der Wiener Publizisten Vincenz Hanzely und Johann Christian Herchenhahn, welche die erste Instanz für Zivilprozesse der kleineren italienischen Reichsvasallen bei der Plenipotenz sehen, von der "sodann an den Reichshofrath appellirt" werde. Für die größeren Vasallen liege die erste Instanz dagegen unmittelbar beim Reichshofrat. Mit seiner Auffassung sieht er sich mit Johann August Reuß, dem Herausgeber der „Merkwürdigen Reichshofrathsgutachten" Heinrich Wilhelm Bergsträsser und Josua Josef von Rieffel, dem Verfasser des Werks „Der Reichshofrath in Justiz- Gnaden- und andern Sachen", einig, die sich alle "mit Recht gegen die eigene Gerichtsbarkeit" der Plenipotenz erklärt hätten. ${ }^{1}$

\footnotetext{
${ }^{1}$ SEIDENSTICKER, Beyträge 108-110, Zitate 109f. Zu Seidensticker vgl. EISENHART, Seidensticker. Eisenhart beurteilt Seidensticker kritisch und meint, dass seinen Schriften „ein bleibender Werth nicht beigemessen werden" könne (ebd.). Vgl. ferner WEGENER, Die staatsrechtliche Stellung.
}

Dass Seidensticker meint, diesen Punkt so betonen zu müssen, und dass es namhafte Juristen gibt, die eine gegenteilige Auffassung vertreten, wirft ein deutliches Licht darauf, dass schon bei den Zeitgenossen des ausgehenden 18. Jahrhunderts erhebliche Unklarheiten über die Reichsgerichtsbarkeit in Italien bestanden. Das betrifft sowohl die Prozesse der Vasallen als auch die der Untertanen, darunter die Appellationsprozesse. Das Bild ist am Beginn des 21. Jahrhunderts kaum klarer geworden, auch wenn seit einigen Jahrzehnten eine Handvoll italienischer, österreichischer und deutscher Forscherinnen und Forscher bestrebt ist, Licht in die Geschichte des frühneuzeitlichen Reichsitalien zu bringen.

Vor diesem Hintergrund kann der folgende Beitrag nicht beanspruchen, ein durch umfangreiche Studien gefestigtes Bild über Norm und Praxis der Appellationen aus Reichsitalien zu zeichnen. Vielmehr muss er sich darauf beschränken, anhand ausgewählter Quellen einige grundlegende Probleme zu skizzieren und zugleich aufzuzeigen, welche Richtung weitergehende Forschungen einschlagen könnten. Nach einem knappen Überblick über die Geschichte Reichsitaliens in der Frühen Neuzeit wird er sich dem eingangs berührten Problem der Stellung der kaiserlichen Plenipotenz im Rahmen der Reichsgerichtsbarkeit zuwenden und sodann anhand einiger ausgewählter Quellen die Situation um die Mitte und in den Siebziger Jahren des 18. Jahrhunderts näher betrachten. 
An die Zusammenfassung der Ergebnisse schließt sich ein Ausblick auf mögliche weitergehende Forschungen an.

\section{Reichsitalien in der Frühen Neuzeit}

Das frühneuzeitliche Reichsitalien ist ein Thema der Geschichtswissenschaft, das die deutsche, österreichische und vor allem italienische Forschung seit einigen Jahren wiederentdeckt hat, das aber immer noch zahlreiche Fragen offen lässt. Dies deckt sich mit dem Befund, dass auch die Zeitgenossen oft nur recht ungenaue Vorstellungen von diesem Rest des mittelalterlichen Regnum Italiae hatten, das bis zu den Friedensschlüssen von Campo Formio (1797) und Lunéville (1801) unter der Lehnshoheit der römisch-deutschen Kaiser stand, obgleich die Nachfolger Karls V. ebenso wie auf die Kaiserkrönung durch den Papst auch auf die Krönung zu italienischen Königen verzichteten und keinen italienischen Königstitel führten. ${ }^{2}$

Unklar war schon der räumliche Umfang Reichsitaliens. Insbesondere konkurrierten über eine Reihe von Gebieten kaiserliche und päpstliche Oberlehnsansprüche, wie beispielsweise über die Lehen der Grafen und Fürsten von Carpegna. ${ }^{3}$ Am spektakulärsten waren im 18. Jahrhundert die Konflikte um Comacchio sowie um Parma und Piacenza. Während Karl VI. 1724 auf das von seinem Bruder Joseph I. 1708 besetzte Comacchio verzichtete,

2 Vgl. im Überblick ARETIN, Reichsitalien; DERS., Das Alte Reich 1, 112-115, 201-208, 310-312; 2, 85-96, 128134, 194-215, 351-380, 458-467; 3, 63-71, 168-171; zu den Institutionen SCHNETTGER, Das Alte Reich und Italien. Trotz ihrer vom Geist des Risorgimento geprägten Stoßrichtung wichtig immer noch die alte italienische Gesamtdarstellung von PUGLIESE, Le prime strette dell'Austria.

${ }^{3}$ Vgl. CARPEGnA FALCONIERI, Die Reichslehen der Grafen und Fürsten von Carpegna. wurden 1718 von der Quadrupelallianz seine Oberlehnsansprüche auf Parma und Piacenza ausdrücklich anerkannt. Allerdings wurden diese nach 1748 faktisch ebenso wenig ausgeübt wie die über die seit 1737 im Besitz des Hauses (Habsburg-)Lothringen befindliche Toskana, die vielmehr die volle Souveränität beanspruchte. Ähnliches galt für die Republik Genua, die seit den 1630er Jahren die kaiserliche Oberhoheit zurückzuweisen bestrebt war und sie nur noch für eine Reihe kleiner Reichslehen anerkannte, die seit dem 16. Jahrhundert in ihren Besitz gelangt waren. ${ }^{4}$ Die übrigen bedeutenderen Fürsten Oberitaliens waren zwar unbestritten kaiserliche Thronvasallen, hatten aber ebenfalls kein Interesse daran, ihrem Oberherrn größere Rechte einzuräumen als unbedingt nötig. Anders war die Lage bei den kleinen Reichsvasallen, die zur Behauptung ihrer Herrschaft gegen ihre mächtigen Nachbarn auf den kaiserlichen Schutz und die Legitimation durch die kaiserliche Belehnung angewiesen waren. Auch sie wünschten außer in Notsituationen üblicherweise keine kaiserliche Intervention in ihren Lehen, waren aber kaum in der Lage, einer solchen Widerstand entgegenzusetzen.

Neben der Differenzierung zwischen den verschiedenen Territorien Reichsitaliens gilt es auch die zeitliche Dimension und die wechselnden Konjunkturen des kaiserlichen Einflusses im Auge zu behalten. Einen Höhepunkt erreichte dieser in der Zeit Karls V. (1519-1556), um unter Ferdinand I. (1556-1564) und seinen Nachfolgern deutlich zurückzugehen. Seit dem ausgehenden 17. Jahrhundert, als die österreichischen Habsburger sich anschickten, ihre spanischen Verwandten in Italien zu beerben, lässt sich ein Wiederaufstieg der kaiserlichen Autorität feststellen. Dieser erreichte in den ersten Jahrzehn-

\footnotetext{
${ }^{4} \mathrm{Zu}$ Comacchio vgl. Göschel, Das ,Bellum Diplomaticum'; zu Parma-Piacenza TAMBORRA, La pace di Aquisgrana; zu Genua ausführlich SCHNETTGER, „Principe sovrano".
} 
ten des 18. Jahrhunderts angesichts der österreichischen Hegemonialstellung auf der Halbinsel seinen Höhepunkt, war aber seit den 1730er Jahren, nach den Niederlagen im Polnischen Thronfolgekrieg und im Österreichischen Erbfolgekrieg, erneut auf dem Rückzug, ohne jedoch bis zum Ausgang des Jahrhunderts ganz zu verschwinden. 5

\section{Die kaiserliche Plenipotenz in Italien}

Mit dem Wiederaufstieg der kaiserlichen Macht in Italien im 18. Jahrhundert war auch eine institutionelle Neuschöpfung verbunden, die für die Reichsgerichtsbarkeit in Italien von erheblicher Bedeutung war: die Plenipotenz in Italien. Schon im Gefolge des Berichts des Reichshofrats Paul Garzweiler über seine Reise in die italienischen Lehen (1603/04) hatte Wien spätestens in den 1620er Jahren versucht, die Kontrolle über die Lehen auf der Apeninnenhalbinsel durch die Ernennung eines dem italienischen Reichsadel entstammenden Generalkommissars zu verstärken, während man sich zuvor fallweise ernannter Kommissare bedient hatte. Nicht zuletzt auf Betreiben des Reichshofrats, der auf die Nutzlosigkeit der Generalkommissare verwies, aber wohl auch einen Einflussverlust in reichsitalienischen Angelegenheiten fürchtete, kehrte man 1639 zum früheren Verfahren der fallweise ernannten Kommissare zurück. ${ }^{6}$

Ein erster Schritt in Richtung auf eine mit dem Wiederaufstieg der kaiserlichen Macht einhergehende institutionelle Verfestigung war 1693

\footnotetext{
${ }^{5}$ Vgl. ARETIN, Das Alte Reich 2, 85-96, 128-134, 194215, 351-380, 458-467; 3, 63-71, 168-171; SCHNETTGER, Abschied von Germania 42-51.

${ }^{6}$ Vgl. ARETIN, Reichsitalien 91-101; RILL, Reichsvikar; Del PINO, Un problema burocratico; SCHNETTGer, Kooperation 129-133; DERS., Das Alte Reich 387-397.
}

die Ernennung eines Reichsfiskals für Italien. ${ }^{7}$ In dieser Zeit deutete sich zudem eine Rückkehr zum Generalkommissariat an, indem immer wieder dieselben Männer zu kaiserlichen Kommissaren berufen wurden, also eine verstärkte Kontinuität in diesem Bereich eintrat. Die Einrichtung der kaiserlichen Plenipotenz erfolgte aber erst mit der Berufung des bereits im kaiserlichen Dienst erprobten Carlo Borromeo Arese zum kaiserlichen Generalkommissar und Plenipotentiar im Jahr 1715. In seiner Instruktion spielte neben zahlreichen anderen Punkten auch das Justizwesen eine wichtige Rolle. Diesbezüglich sollte Borromeo Sorge tragen, dass die Reichsvasallen ihren Untertanen Justiz leisteten und ihnen nicht den Weg zu Beschwerden oder förmlichen Appellationen an den Reichshofrat abschnitten. ${ }^{8}$ Borromeo wurde also eine Oberaufsicht über die Justiz der Reichsvasallen zugesprochen, wobei das Offenhalten der Appellationsmöglichkeit nach Wien ein wichtiges Anliegen darstellte. Was Borromeo hingegen nicht erhielt, waren eigene jurisdiktionelle Kompetenzen. Ihm wurde zwar in der Person Johann Jacob Joanellis ebenfalls ein Reichsfiskal beigegeben, doch eine eigene gerichtliche Instanz bildete die Plenipotenz nicht. Vielmehr nahm sie

\footnotetext{
${ }^{7}$ Der erste italienische Fiskal war Graf Mezzabarba Birago. Vgl. zu seiner Berufung und allgemein zum italienischen Reichsfiskalat OBERSTEINER, Das Reichshoffiskalat.

8, ",...] quatenus omni studio in eo sit, ut subditi vasallis debitam obedientiam præstent, et hi vicissim subditis justitiam rectè administrent, nec illos plus æquo onerent, neque impediant, quominùs justas oppressionum suarum querelas ad Nos vel commissarium nostrum imp[erial]em et plenipotentiarium deferant, aut in causis judicialibus ad consilium nostrum, imp[erial]e aul[i]cum appellent, nisi fortè vasallus legitimo privilegio de non appellando, cui nihil derogare volumus, ritè munitus esset“. Instruktion für Carlo Borromeo Arese, Wien, 24. 4. 1715, HHStA Wien, RHR, Plenipotenz 3, Fasz. 1707-1716, fol. 98105, hier: $103^{v}$.
} 
im Wesentlichen die Aufgaben einer ständigen Untersuchungskommission für Italien wahr. ${ }^{9}$

Detailliertere Anweisungen zum Thema Appellation finden sich in der Instruktion für den Reichsfiskal Christoph Werth (1722-1736). Unter Bezugnahme auf ein Reskript an den Plenipotentiar aus dem Jahr 1721 wird Folgendes vorgeschrieben: Ein Prozess gegen einen Untertan eines Vasallen, der über die Straf- und Zivilgerichtsbarkeit verfügt, solle am erstinstanzlichen Gericht dieses Vasallen geführt werden. Wenn sich aber eine der Parteien durch das Urteil graviert glaube, stehe es ihr frei, durch Appellation oder ein anderes Rechtsmittel den Weg eines Rekurses an den Kaiser zu beschreiten. In allen diesen Fällen wurde auch die Bitte um eine Vergleichsverhandlung vor dem Plenipotentiar binnen Monatsfrist zugelassen, dem somit zwar ausdrücklich eine Vermittlungsfunktion in Appellationsfällen, aber eben keine Rechtsprechungskompetenz zugestanden wurde..$^{10}$

\footnotetext{
9 Vgl. SCHNETTGER, Kooperation 133-149 (mit Verweisen auf die ältere Literatur).

${ }^{10},[\ldots]$ ut si vasallus Imperialis cum mero et mixto Imperio investitus sit, tunc causam, in qua contra talis vasalli subditum actio instituitur, ad primam instantiam remittat, salva semper manente libertate, ut, si quae partium per sententiam in prima hujusmodi instantia latam se ex motivis relevantibus putet gravatam esse, per appellationem aut aliud legale remedium servatis servandis, ad majestatem Nostram Caesaream humillime recurrere possit in omnibus tamen casibus, in quibus, modo dicto, ad Nos a sententia primae instantiae recurritur, clementissime permittimus ut parti, quae se illa gravatam putat, amicabilem autem transactionem cum parte adversa inire cupit, post appellationem intra decendium legaliter interpositam, adhuc intra mensem Nostrum Caesareum in Italia plenipotentiarium commissarium pro causa amicabiliter componenda adire liceat, ita tamen, ut interponendae appellationis et reliqua fatalia in omnem eventum circa sententiam primae instantiae observare teneatur". Instruktion für den kaiserlichen Fiskal in Italien Christoph Werth, 29.4. 1724, zit.n. OBERSTEINER, Reichshoffiskalat (Staatsprüfungsarbeit) 112-115, hier: 114 .
}

\section{Der Vorstoß des Grafen Stampa im Jahr 1751 und die Reaktionen des Reichshofrats ${ }^{11}$}

Die Plenipotentiare waren vorhersehbar bestrebt, ihre Kompetenzen im Bereich der Rechtsprechung auszubauen. Dies trifft insbesondere auf den Neffen und unmittelbaren Nachfolger Borromeos Carlo Stampa (1734-1740, 1745-1753) zu, der schon während der Amtszeit seines Onkels einige Einblicke in die Arbeit der Plenipotenz und deren Probleme getan hatte. Stampa erhielt bei seinem ersten Amtsantritt aufgrund der Wirren des Polnischen Thronfolgekriegs keine eigene Instruktion, sondern wurde lediglich auf diejenige seines Vorgängers verwiesen, doch zu Beginn seiner zweiten Amtszeit gab es ausführliche Beratungen über die Stellung des Plenipotentiars, bei welchen auch Überlegungen Stampas aus den 1730er Jahren erörtert wurden, die auf eine deutliche Stärkung seiner Rechtsprechungskompetenzen hinausliefen. Auch später bemühte sich Stampa immer wieder um eine Ausweitung seiner Jurisdiktionsrechte, stieß dabei aber regelmäßig auf den Widerstand des Reichshofrats, so etwa im Zusammenhang mit der Formulierung der Instruktion für den neuen italienischen Reichsfiskal Rath, die an die Bestimmungen von 1724 anknüpfte, allerdings eine wichtige Änderung vornahm: In Paragraph 29 wurde nicht nur allgemein verboten, einen Rekurs an den Kaiser zu verhindern, sondern ausdrücklich untersagt, dass die Vasallen zum Schaden der kaiserlichen Jurisdiktionsrechte selbst Appellationsrichter bestimmten. Dies lief darauf hinaus, den Vasallen (sofern sie nicht im Besitz eines Appellationsprivilegs waren) lediglich die erste Instanz zuzugestehen. Außerdem ergänzte die Instruktion einen Paragraphen zu Fällen von Rechtsbeugung. Auch hier sollte der

\footnotetext{
${ }^{11}$ Vgl. zum Folgenden ARETIN, Reichsitalien 97f.; DERS., Das Alte Reich 3, 64f.
} 
Plenipotentiar keine eigentliche Rechtsprechungsgewalt besitzen, durfte aber immerhin unter Hinzuziehung des Fiskals entscheiden, ob die Beschwerde der rekurrierenden Partei zu Recht erfolgt sei. In diesem Fall sollte er nach Wien Bericht erstatten; wobei unterdessen das Urteil des betreffenden Vasallen oder seines Richters zu suspendieren war. Wenn Vasall und Richter hingegen nachweisen konnten, nach Recht und Gesetz vorgegangen zu sein, sollte der Beschwerdeführer unverzüglich an diese zurückverwiesen werden. ${ }^{12}$

Im Jahr 1751 wagte Stampa einen neuen Vorstoß zur Ausweitung seiner Rechtsprechungskompetenzen. Im Mai und November dieses Jahres verabschiedete der Reichshofrat in diesem Zusammenhang zwei umfangreiche Voten an Kaiser Franz I., die im Folgenden ausführlich wiedergegeben werden sollen, da sie einige grund-

\footnotetext{
12 "Vigesimo nono. Nullus autem vasallorum ejusmodi recursus (nisi forsan legitimo et speciali privilegio de non appellando gaudeat) impedire, aut post suam vel praetoris sententiam in praejudicium supremae Nostrae jurisdictionis Caesareae judicem appellationis designare et constituere conetur. [...] Trigesimo secundo. Imprimis vero eo invigilandum erit, ut hisce causis ex omni parte a vasallis eorundemque judicibus legaliter procedatur, idcirco si quis in feudis Nostris Imperialibus ex capite nullitatis insanabilis vel manifestae iniquitatis ad plenipotentiarium Nostrum recurrat, eundem ad audientiam admitti, adhibita ope ficalis Nostri Caesarei, allegatam nullitatem vel iniquitatem probe examinari, et hoc praevio si justa conquerendi causa subsit, Nobis a plenipotentiario Nostro casum, annexo voto suo humillimo, referri volumus. Suspensa interim feudatarij aut judicis sententia sub poena mulctae pecuniae quinque marcarum auri Caesareo Nostro fisco applicandae et authoritate Caesarea per plenipotentiarium Nostrum iisdem intimandae. Econtra si ex petitis a vasallo vel judice informationibus appareat, eosdem intramite recto juris et justitiae processisse, recurrentem sine mora ad illos pro sententiae executione remitti clementissime jubemus". In den Paragraphen 28 und 30 der Instruktion werden die genannten Verfügungen von 1724 wiederaufgegriffen. Instruktion für den kaiserlichen Fiskal in Italien Anton Eberhard Rath, 28.6. 1748, zit.n. OBERSTEINER, Reichshoffiskalat (Staatsprüfungsarbeit) 116-122, hier: $119 f$.
}

sätzliche Probleme im Zusammenhang mit der Gerichtsbarkeit in Reichsitalien und auch mit den Appellationen beleuchten, ja einen Höhepunkt in den Konflikten zwischen Wiener und italienischen Reichsbehörden um die Rechtsprechung in Italien markieren.

Wie üblich, beginnt das Reichshofratsgutachten vom Mai 1751 mit einer ausführlichen Schilderung der Sachlage, wie sie sich gemäß den Berichten des Plenipotentiars darstelle. Dabei werden folgende Einzelfragen erörtert:

Unter Berufung auf ein Reskript Karls VI. an seinen Vorgänger Borromeo aus dem Jahr 1714, also vor dessen Ernennung zum Plenipotentiar, beansprucht Stampa ohne Weiteres die Rechtsprechung über die Vasallen in Fällen von geringer Bedeutung und dort, wo Gefahr im Verzug ist. Da aber die Vasallen dazu tendieren, die Rechtsprechungskompetenzen des Plenipotentiars in Zweifel zu ziehen, es sei denn sie wäre ihm vorher für einen konkreten Fall vom Kaiser übertragen worden, empfiehlt Stampa, die Regelung von 1714 so zu verändern, dass grundsätzlich alle Fälle der Jurisdiktion des Plenipotentiars unterliegen sollen mit Ausnahme derjenigen, die von erheblicher Bedeutung seien und wo keine Gefahr im Verzug sei. Über diese Fälle werde er an den Kaiser berichten. Es geht Stampa also im Wesentlichen darum, die bisherige Ausnahme zur Norm zu machen, wobei er für sich zugleich die Vollmacht beansprucht festzulegen, welche Fälle nach Wien zu referieren sind. Dies werde, so seine Argumentation, dazu dienen, die Rechtsprechung gerade auch für die Armen zu beschleunigen.

Der Reichshofrat spricht sich jedoch mit Vehemenz dagegen aus, diesem Ansinnen zu entsprechen und erinnert daran, dass er dem schon 1748, im Zuge der Beratungen über die Instruktion des italienischen Fiskals, widerraten habe, da „die wichtigkeit deren von zeit zu zeit vorfallenden objectorum, und dabey interessirten persohnen allerdings erfordere, mit allergrösten vorsicht, und behutsambkeit zu verfahren". 
Außerdem würde "die übertragung einer gerichtlichen gewalt bey denen welschen vasallen ein großes aufsehen erwecken, und mit der grund-verfassung der kay[serlichen] plenipotenz in Italien /: welche bißhero lediglich in terminis commissionis caesareae, nicht aber eines judicii formati bestanden:/ nicht wohl übereinstimmen“. Die Autorität des Plenipotentiars werde durch die bestehende Regelung nicht beeinträchtigt: Falls seinen Anordnungen nicht Folge geleistet werde, könne er sich ja an den Kaiser wenden. Daher sollte Stampa darauf hingewiesen werden, dass Vasallen und Untertanen freistehen solle, ihren Rekurs an den Kaiser oder an den Plenipotentiar zu nehmen. Allerdings sollte Letzterer nach wie vor lediglich als Vermittler tätig werden und im Falle eines Scheiterns der Mediation an den Kaiser berichten, statt eigenmächtig einen Prozess zu führen. Wenn Gefahr im Verzug sei oder ein irreparabler Schaden drohe, dürfe er die ihm notwendig erscheinenden Maßnahmen ergreifen, jedoch nur provisorisch und unter Berichtspflicht an den Kaiser. ${ }^{13}$

Eine zweite Forderung Stampas richtet sich darauf, dass die Plenipotenz die erste Instanz für Streitfälle zwischen den Vasallen und ihren Untertanen bilden solle, was der bisherigen Praxis entspreche und so schon in der Instruktion des Fiskals enthalten sei. Abermals jedoch widerspricht der Reichshofrat und wendet vielmehr ein, dass Stampa dieses Ansinnen zwar schon bei den Beratungen über die Instruktion des Fiskals geäußert habe, dass dieser Passus seinerzeit aber eben nicht in die Instruktion übernommen worden sei, vor allem aus dem Bestreben heraus, Vasallen und Untertanen nicht den unmittelbaren Rekurs an den Kaiser zu versperren. Aus diesem Grund und wiederum um

\footnotetext{
${ }^{13}$ Reichshofratsgutachten, conclusum, lectum et approbatum 6. 5. 1751, resolviert vom Kaiser 28. 5. 1751, HHStA Wien, RHR, Plenipotenz 5, Fasz. 1, fol. 351 $376^{v}$, hier: $351^{\mathrm{v}}-357^{\mathrm{v}}$, Zitat $355^{\mathrm{r}}$.
}

grundsätzlich nicht der Plenipotenz den Charakter eines ordentlichen Gerichts beizulegen, spricht sich der Reichshofrat erneut gegen diese Forderung Stampas aus. ${ }^{14}$

Das dritte Anliegen Stampas betrifft direkt das Problem der Appellationen. Hierzu referiert das Reichshofratsgutachten folgenden Sachverhalt: Der Plenipotentiar habe auf einen Missbrauch hingewiesen, der sich seit langem in den Reichslehen eingebürgert habe, nämlich dass die Vasallen bei einer Appellation gegen ein Urteil ihrer Niedergerichte selbst die Appellationsrichter bestimmten. In der Instruktion des Fiskals sei ausdrücklich enthalten, dass die Vasallen in Prozessen gegen ihre Untertanen keine Appellationsrichter benennen dürften, sondern den Untertanen der Rekurs an den Kaiser oder zu einer Vergleichsverhandlung vor dem Plenipotentiar offenstehen müsse. „Hiebey entstehe aber die gröste difficultät in deme, daß die die [!] welschen Reichs-Vasallen in observantia immemoriali sich befindeten, ejusmodi judices appellationis zu constituiren“ und "sogar dießfalls statuta" hätten. Zudem würde es den Untertanen schwerfallen, wegen eines Streitwerts von einigen Gulden nach Wien zu appellieren oder einen Prozess vor der Plenipotenz in Pisa zu führen. Es wäre also damit zu rechnen, dass Vasallen und Untertanen einhellig gegen eine solche Neuregelung der Appellationen protestieren würden. Wenn also der Kaiser beabsichtige, den Vasallen nur eine Instanz zuzugestehen, bleibe nur das Mittel, dass in den Lehen oder deren Nachbarschaft ein kaiserlicher Appellationsrichter eingesetzt werde, „weilen man doch die unterthanen ohne appellation nicht lassen könne“. Falls Franz I. dagegen den Vasallen die ordnungsgemäße Bestellung eines Appellationsrichters zugestehen wolle, möge er dem Plenipotentiar gestatten, im Beschwerdefall „denen an ihn recurrirenden subditis pro diver-

${ }^{14}$ Ebd. fol. 357v-359v. 
sitate causarum et situationis feudorum einen schicklichen in der nähe sich befindenden judicem appellationis $\mathrm{zu}$ constituiren" - jedoch stets vorbehaltlich der Möglichkeit des Rekurses an den Kaiser. ${ }^{15}$

In seinem Votum unterstreicht der Reichshofrat die von Stampa angeführten Probleme und vermutet mit gutem Grund, dass der Plenipotentiar deswegen die in der Instruktion des Fiskals enthaltenen Bestimmungen bezüglich der Appellationen noch nicht publiziert habe. Es sei zwar richtig, dass die Paragraphen 28 und 29 der Instruktion die skizzierten Bestimmungen bezüglich der Appellation enthielten, doch der neue Paragraph 29 gehe auf die Eingaben Stampas und Raths selbst zurück und wäre sicher nicht so ausgefallen, wenn die nun angeführten Schwierigkeiten seinerzeit schon geäußert worden wären. „Eine observantiam immemorialem (wann sie an sich gleich etwas irregulaires enthaltet) sogleich de simplici et plane aufzuheben", sei "allezeit bedenklich". Da aber in fast ganz Italien „der modus procedendi usque ad tres conformes sententias herkomlich" sei, also mehrere Instanzen „coràm judice inferiore erlaubet seynd“, würde es im aktuellen Fall darum gehen, „eine observantiam vix non universalem aufzuheben“. Daher empfahl der Reichshofrat dem Kaiser, das gebräuchliche Appellationsverfahren grundsätzlich beizubehalten, jedoch vorbehaltlich des Rekurses an den Kaiser in letzter Instanz. Auch wenn jemand direkt von der ersten Instanz an das Reichsoberhaupt appellieren wolle, dürften das die Vasallen nicht verhindern. Das in der Instruktion des Fiskals enthaltene grundsätzliche Verbot der Bestimmung von Appellationsrichtern durch die Vasallen empfahl der Reichshofrat also zurückzunehmen oder doch einzuschränken. Eine klare Absage erteilte der Reichshofrat dem ,abermahlen auf eine extension der einem zeitlichen

\footnotetext{
${ }^{15}$ Ebd. fol. 360-362, Zitate fol. 361 ${ }^{\mathrm{r}}, 362^{\mathrm{r}}$.
}

Kay[serlichen] plenipotentiario zustehenden macht" abzielenden Vorschlag Stampas, ihm die Vollmacht zu erteilen, bei Bedarf selbst einen Appellationsrichter $\mathrm{zu}$ bestimmen. Stattdessen solle er in einem solchen Fall die Entscheidung des Kaisers einholen. ${ }^{16}$

Bezüglich der von Stampa allgemein angesprochenen verschiedenen Unregelmäßigkeiten im Gerichtswesen der Vasallen sah sich der Reichshofrat nicht zu einer Stellungnahme in der Lage, bevor nicht der Plenipotentiar und der Fiskal einen ausführlichen Bericht und ihre diesbezüglichen Voten vorgelegt hätten. Ferner ging es in dem Reichshofratsgutachten um einige konkrete Rechtsfälle, die zum Teil mit dem Appellationsproblem im Zusammenhang standen, hier aber nicht weiter betrachtet werden können. ${ }^{17}$

Da Franz I. am 28. Mai dem Reichshofratsvotum in allem zustimmte, dürfte das auf dieser Basis formulierte kaiserliche Reskript für Stampa eine herbe Enttäuschung bedeutet haben. ${ }^{18}$ Der Pleni-

${ }^{16}$ Ebd. fol. 362r $-365^{\mathrm{v}}$, Zitat fol. 364 ${ }^{\mathrm{r}}, 365^{\mathrm{r}}$.

${ }^{17}$ Die Appellationsfälle, die Graf Stampa in seinen Berichten des Jahres 1751 anführte, betrafen mindermächtige Reichsvasallen, wie insbesondere den Marchese Cornelio von Liciana aus dem Haus Malaspina, und deren Untertanen. Häufig ging es hierbei in der Tat um geringe Streitwerte. So prozessierten Alderico Guerrieri und seine Ehefrau Margherita, geb. Bruschi, um die Abgabenfreiheit einiger Güter, die sie in Liciana von ihrem Vater respektive Schwiegervater Leonardo Bruschi geerbt hatten. Derselbe Guerrieri lag zugleich in einem Rechtsstreit mit den Brüdern Ridolfi, die ihm ein ziemlich heruntergekommenes Haus verkauft hatten, das sie aber, nachdem Guerrieri es hatte wieder in Stand setzen lassen, zurückforderten. Reichshofratsgutachten, conclusum, lectum et approbatum 6. 5. 1751, resolviert vom Kaiser 28.5. 1751, HHStA Wien, RHR, Plenipotenz 5, Fasz. 1, fol. 351 ${ }^{\mathrm{r}}$ 376 ${ }^{v}$, hier: 366-375. Cornelio Malaspina hatte den Zorn des Plenipotentiars außer durch Verfahrensfehler in den genannten Appellationsfällen auch dadurch erweckt, dass er die Titulatur "Nos Dei gratia“ verwendet hatte, ebd. fol. $375^{r-v}$.

${ }_{18}$ Dieses enthielt zudem einen deutlichen Verweis an Stampa, er möge künftig in seinen Schreiben nicht verschiedene Materien vermischen, sondern alle Ge- 
potentiar blieb jedoch hartnäckig und wagte in Abstimmung mit dem italienischen Reichsfiskal noch im September desselben Jahres einen erneuten Vorstoß in Wien, indem er den Entwurf eines Edikts an die Vasallen zur Umsetzung des Befehls vom Mai nebst einem entsprechenden Bericht des Reichsfiskals übersandte und um eine diesbezügliche Weisung bat. Unter anderem sprach Rath das Problem an, dass das kaiserliche Reskript keine befriedigende Regelung für Appellationen von Minderbemittelten und bei geringen Streitwerten enthalte, da in solchen Fällen der Weg an den Kaiserhof nicht praktikabel sei. ${ }^{19}$ Mit Blick auf die Armut auch vieler Vasallen malte der Fiskal zudem die Gefahr an die Wand, dass diese zur gewaltsamen Selbsthilfe greifen würden, wenn es ihnen an Mitteln fehle, in Wien zu prozessieren. ${ }^{20}$

Schließlich wies Rath auf die Konsequenzen der mangelnden Entscheidungsgewalt des Plenipotentiars hin, dem nach dem Scheitern einer Vergleichsverhandlung und wenn der Kläger nicht in Wien prozessieren könne, nur übrigbleibe, die Akten an den Kaiser zu versenden und um die Übertragung der Untersuchung in dem betreffenden Fall zu ersuchen. Damit wäre aber weder den Parteien gedient, denen an einer zügigen Entscheidung gelegen sei, noch der Plenipotenz, deren Ansehen sehr darunter leiden würde, noch der Reichshofkanzlei und dem Reichshofrat, die auf diese Weise mit Arbeit überhäuft würden. Daher möge der Kaiser dem Plenipotentiar in den Fällen minderbemittelter Kläger auf deren Wunsch ohne vorherige Spezialdelegation die Rechtsprechung übertragen oder einen anderen praktikablen Weg aufzeigen,

genstände gesondert abhandeln und seinen Berichten in Justizangelegenheiten zugleich das Votum des Fiskals anfügen, ebd. fol. $375^{\mathrm{v}}-376^{\mathrm{r}}$.

${ }^{19}$ Reichshofratsgutachten, conclusum, lectum et approbatum 6.11. 1751, HHStA Wien, RHR, Pleni-

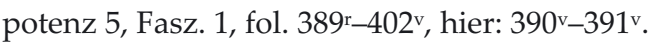

${ }^{20}$ Ebd. fol. 392v. wie man in solchen Fällen verfahren solle. ${ }^{21}$ Schließlich legte Rath das Konzept eines gedruckten Edikts zur Justiz in Reichsitalien bei, von dem jeweils zwei Exemplare in jedem Lehen hinterlegt werden sollten. ${ }^{22}$

In seinem Votum wies der Reichshofrat die Anträge des Plenipotentiars und des italienischen Fiskals schroff zurück. Stampas „versteckte absicht" gehe "abermahlen dahin, sich, in vasallos Italiae eine größere macht beyzulegen, und gegen das institutum der Kay[serlichen] plenipotenz aus derselben ein förmliches judicium formatum zu machen", wogegen sich Reichshofrat und Kaiser schon im Mai mit Bedacht ausgesprochen hätten. „Alles, was der fiscal in seiner relation pro amplianda jurisdictione plenipotentiarii Caes[arei] anziehet", enthalte "weder etwas neues, noch erhebliches", und das Interesse der Parteien an einem zügigen Prozess wolle „nicht viel bedeuthen, wann dargegen die besorgliche inconvenienzien gehalten werden, welche leicht entstehen könnten, wann es dabey auf das merum arbitrium eines zeitlichen commissarii ankommen sollte". Auch das Ansehen der Plenipotenz werde dadurch nicht beschädigt, da diese bekanntermaßen "die judicialia [...] nur accessoriè zum gegenstand" habe. ${ }^{23}$

Ebenso sprach sich der Reichshofrat gegen die Publikation des Edikts in der vorgelegten Form aus, denn durch dessen „affixion in omnibus indistinctè feudis Imperii Italiae“ könnte "bey denen höheren vasallen ein großes aufsehen erwecket werden $[\ldots]$, welches Euer Kay[serlichen] May[estät] authorität denen heütigen umständen nach bey einem oder andern vielleicht allerhand bedencklichen folgen exponiren dörffte, weilen durch die letzte Kay[serlich]e verordnung in materia appellationum die bißherige observantia immemoralis constituendi plures judices appellationis als ein abusus abge-

\footnotetext{
${ }^{21}$ Ebd. fol. 394 ${ }^{\mathrm{r}-\mathrm{v}}$.

${ }^{22}$ Ebd. fol. 394v.

${ }^{23}$ Ebd. fol. 395-398, Zitat fol. 395r , 396rv , 397r .
} 
stellet, und diese facultas vasallorum [...] lediglich auf dem fall, wann die partheyen damit zu frieden seynd, restringiret worden". Die bisherige Appellationspraxis wollte der Reichshofrat also nicht umstoßen. Lediglich gegen eine Publikation der Prozessordnung bei der Plenipotenz hatte der Reichshofrat keine Bedenken. ${ }^{24}$

Insgesamt wird deutlich, dass der Reichshofrat einerseits bestrebt war, den Untertanen und mindermächtigen Vasallen die Möglichkeit eines Rekurses oder einer Appellation an den Kaiser als obersten Richter und Herrn offenzuhalten. Andererseits schreckte er davor zurück, die bestehenden Strukturen in Reichsitalien radikal zu verändern und war ihm daran gelegen, die Kompetenzen der Plenipotenz in Justizfragen strikt zu begrenzen und diese v.a. nicht zu einer ordentlichen Instanz auszubauen - und das hatte unvermeidlich auch Konsequenzen für Norm und Praxis der Appellationen aus Italien.

\section{Die Diskussion in den 1770er Jahren}

Das Thema der Jurisdiktionsrechte der Plenipotenz und in diesem Zusammenhang ihre Stellung als Appellationsinstanz wurde erneut in den 1770er Jahren diskutiert, in einer Zeit, als Joseph II. Kaiser war. Dieser zeigte insbesondere in den Jahren vor der Übernahme der Alleinregierung in Österreich ein großes Interesse an der Reichsjustiz, betrieb mit viel Engagement (wenngleich begrenztem Erfolg) die Visitation des Reichskammergerichts und bemühte sich auch um die Abstellung von Missbräuchen beim Reichshofrat. ${ }^{25}$ Ebenso lag ihm die Justiz in der österreichischen Lombardei am Herzen. ${ }^{26}$

Auch hinsichtlich der Reichsgerichtsbarkeit in Italien zeigte Joseph II. ein beachtliches Enga-

\footnotetext{
${ }^{24}$ Ebd. fol. 398-402, Zitat fol. 400r-401v.

${ }^{25}$ Vgl. ARETIN, Das Alte Reich 3, 135-165.

${ }^{26}$ Vgl. Petronio, Il Senato di Milano 370-427.
}

gement. Er war hier grundsätzlich bestrebt, die Reichsrechte $\mathrm{zu}$ behaupten und womöglich auszubauen, was ihn unter anderem in Konflikt mit der Republik Genua brachte. Hier ging es mit den Fällen Campo freddo und San Remo um zwei Extrajudizialappellationen von europäischer Bedeutung, umso mehr als Genua sich den Schutz des Allerchristlichsten Königs zu verschaffen wusste. Infolge des Vorpreschens Josephs II. im Fall San Remo kam es 1766/67 zu einem Eklat und $\mathrm{zu}$ einer schweren Verstimmung zwischen Wien und Versailles, die den jungen Kaiser zu einem blamablen Rückzug nötigte. Diese Affäre verdeutlichte, wie leicht es in Reichsitalien $\mathrm{zu}$ einer Vermischung von Rechtsprechung und großer europäischer Politik kam und dass in solchen Fällen üblicherweise die Belange der Reichsjustiz hinter die Interessen der Großmacht Österreich zurückzutreten hatten. ${ }^{27}$ Derartige Rücksichten dürften auch die Bereitschaft in Wien gehemmt haben, wegen einer Reform der Justiz in Reichsitalien Konflikte von unkalkulierbaren Dimensionen zu riskieren. Immerhin kam es zu einer Reihe von strukturellen Veränderungen. So wurde nach dem Regierungsantritt Großherzog Peter Leopolds in der Toskana die Plenipotenz in Pavia wiedervereinigt, nachdem sich während der Amtszeit des Plenipotentiars Antoniotto Botta Adorno (1754-1774), der gleichzeitig Statthalter der Toskana für Kaiser Franz I. gewesen war, nicht nur der Plenipotentiar selbst, sondern auch ein Teil des Archivs in Florenz befunden hatten, was die Arbeit dieser Behörde wesentlich erschwert haben dürfte. ${ }^{28}$

Das Problem des Instanzenzugs in Reichsitalien kam im Zusammenhang mit der Bestellung des neuen italienischen Reichsfiskals, Joseph Aloys Leporini (1772-1775), zur Sprache. Dieser griff in einem Memorandum zu der ihm zu erteilenden

\footnotetext{
${ }^{27} \mathrm{Zu}$ den Fällen Campo freddo und San Remo vgl. ausführlich SCHNETTGER, „Principe sovrano“ 334-412. ${ }^{28}$ Vgl. SchnetTGer, Das Alte Reich $395 f$.
} 
Instruktion die in den 1750er Jahren erörterten Probleme auf. Gleich als ersten der zu klärenden bzw. zu ändernden Punkte nannte er die Frage, ob nicht den Untertanen in den italienischen Reichslehen die Appellation an die Plenipotenz gestattet werden solle. Zwar habe die Instruktion seines Vorgängers Rath klar bestimmt, dass die Untertanen (unter Beachtung der Appellationsformalien) lediglich binnen eines Monats eine Vergleichsverhandlung vor der Plenipotenz versuchen dürften. Die in der ersten Instanz erfolgreiche Partei werde sich jedoch hierzu kaum bereitfinden, und sie dazu zu zwingen, werde schwerlich möglich sein. Die Appellation an den Reichshofrat falle der unterlegenen Partei hingegen "allzu beschwerlich [...], weilen solche gemeiniglich sehr arm und der Weg nacher Wien für sie gar zu weit ist"; zudem gehe es meist um nur geringe Streitwerte, für die ein kostspieliger Reichshofratsprozess die Mühe nicht lohne. Als einen Mittelweg und „etwas kräfftigeres remedium“ regte Leporini an, „den armen und öffters unter einem schweren joch der feudatarien eusserst bedrangten Unterthanen" zumindest bis zu einem bestimmten Streitwert die Appellation an die Plenipotenz zu gestatten (,,alß wohin sie mit einem stuck brod im Rantzen lauffen können“), die dann eine gütliche Einigung versuchen solle. Der Unterschied des Vorschlags zur bisherigen Regelung war nur gering; er lief aber darauf hinaus, die Befugnisse der Plenipotenz als reguläre Appellationsinstanz wenn auch mit begrenzter Entscheidungskompetenz zu stärken..$^{29}$

Eine klare Entscheidung erbat Leporini auch in der von ihm als bisher offen dargestellten Frage, ob die italienischen Vasallen befugt seien, ihren Untertanen nach den Entscheidungen der ersten

${ }^{29}$ Memorial des italienischen Reichsfiskals Joseph Aloys Leporini, undat. [1772], HHStA Wien, RHR, Plenipotenz 5, Fasz. 1752-1766, fol. 354-367, hier: 354ㄴ-355v. Vgl. AREtin, Das Alte Reich 3, $169 f$.
Instanz einen Appellationsrichter zu benennen. ${ }^{30}$ Damit hing auch das nächste Problem zusammen, nämlich ob von der ersten oder zweiten Instanz aus die Appellation an Reichshofrat oder Plenipotenz (je nach Höhe der Appellationssumme) erlaubt sein solle. ${ }^{31}$ Zwar war der Fiskal bereit, zumindest einem Teil der Vasallen das bisher praktizierte Recht einzuräumen, den Richter der zweiten Instanz selbst zu bestimmen. Er stellte es aber als selbstverständlich hin, dass die dritte Instanz je nach Streitwert der Reichshofrat oder die Plenipotenz sein müsse.

Die Instruktion für den neuen Plenipotentiar Johann Sigismund Fürst Khevenhüller-Metsch hielt an der Anschauung fest, dass erhebliche Gründe gegen die Erhebung der Plenipotenz zu einem ordentlichen Gericht sprächen, erinnerte an die Auseinandersetzungen in den frühen 1750er Jahren und verwies ausdrücklich auf die seinerzeitigen Reskripte an Stampa. Es wurde Khevenhüller-Metsch nur (gemäß der bestehenden Praxis) zugestanden, Gravamina derjenigen, die aufgrund ihrer Armut oder wegen der großen Distanz nicht ihren direkten Rekurs an den kaiserlichen Hof nehmen könnten (,recurrere nequeunt"), nach Wien zu melden und die kaiserliche Entscheidung zu erwarten. ${ }^{32}$ Bezüglich des Schutzes derjenigen, die sich nicht an den Kaiser wenden könnten, vor Unterdrückung, Rechtsbeugung und Rechtsverweigerung und hinsichtlich der Ad-hoc-Maßnahmen, um plötzlichen Gefahren zu begegnen, verwies die In-

\footnotetext{
${ }^{30}$ Leporini bezieht diese Frage auf die mit dem „merum et mixtum imperium" ausgestatten Vasallen, legt also nahe, dass die anderen dieses Recht wohl nicht besäßen. Memorial des italienischen Reichsfiskals Joseph Aloys Leporini (wie Anm. 29) fol. 355 $-356^{\mathrm{r}}$. ${ }^{31}$ Ebd. fol. 356.

${ }^{32}$ (Zweite) Instruktion für Johann Sigismund Fürst Khevenhüller-Metsch, Wien, 15.9. 1775, HHStA Wien, RHR, Plenipotenz 6, Fasz. 1775-1792, fol. 106 $114^{v}$, hier: 111v. Zugeständnis in dem Reichshofratsgutachten zu dieser Instruktion, 22. 8./1. 9. 1775, ebd. fol. 6-25.
} 
struktion ebenfalls auf die Reskripte von 1751, stellte Khevenhüller-Metsch aber frei, etwaige Verbesserungsvorschläge dem Kaiser zu unterbreiten, der sodann darüber befinden werde. ${ }^{33}$

Alles in allem zeigte sich der Reichshofrat auch in den 1770er Jahren wenig geneigt, der Plenipotenz eigenständige Rechtsprechungsbefugnisse zuzugestehen, und Joseph II. scheint dieser Auffassung gefolgt zu sein. Während man grundsätzlich den Untertanen und kleinen Vasallen den Rechtsweg an den Reichshofrat offenhalten wollte, beschränkte man die Kompetenzen der Plenipotenz nach wie vor auf die eines Mediators. Dass Appellationen an die Reichsgerichte Vasallen und Untertanen grundsätzlich offenzustehen hätten, war dagegen in der Reichsadministration nicht umstritten. Man lehnte es also ab, die Appellationsverfahren ganz in die Hände der Vasallen zu geben. Eine grundsätzliche Erweiterung der Zuständigkeiten der Plenipotenz lässt sich jedoch nicht nachvollziehen. Vielmehr erhielt der letzte Plenipotentiar Johann Joseph Maria Graf Wilczek, der 1782 nach einem kurzen Intermezzo, in dem Karl Graf Firmian das Amt verwaltete, ernannt wurde, gar keine eigene ausführliche Instruktion mehr, sondern wurde im Wesentlichen auf die seines Vorgängers verwiesen. Zudem war er ebenso wie Firmian zugleich und vor allem Bevollmächtigter Minister in der österreichischen Lombardei. ${ }^{34}$ Wie weit das von Aretin berichtete Zugeständnis Josephs II. aus dem Jahr 1786 trug, die Plenipotenz zur zweiten Instanz in Armensachen zu erklären, konnte im Rahmen dieses Beitrags nicht verfolgt werden. ${ }^{35}$

\footnotetext{
${ }^{33}$ (Zweite) Instruktion für Johann Sigismund Fürst Khevenhüller-Metsch (wie Anm. 32) fol. 111v-112 .

${ }^{34}$ Instruktion für Johann Joseph Maria Graf Wilczek, Wien, 26. 6. 1782, HHStA Wien, RHR, Plenipotenz 6, Fasz. 1775-1792, fol. 116-119. Vgl. SCHNETTGER, Das Alte Reich 396.

35 ARETIN, Reichsitalien 101. Leider bietet Aretin an dieser Stelle keinen Nachweis für seine Aussage. In Das Alte Reich 3, 170, spricht ARETIN allgemeiner
}

\section{Schluss}

Die italienischen Appellationsprozesse sind im weiteren Kontext der Reichsgerichtsbarkeit in Italien zu betrachten. Diese hatte im Allgemeinen und gerade auch im Zusammenhang mit Appellationsprozessen mit besonders schwierigen Rahmenbedingungen zu kämpfen. Der Grundsatz der kaiserlichen Höchstgerichtsbarkeit in Reichsitalien war für Reichshofrat und Plenipotentiar unantastbar, denn das oberstrichterliche Amt gehörte zu den vornehmsten Aufgaben und Kompetenzen des Reichsoberhaupts. Daher musste der Rechtsweg an den Kaiser jedem italienischen Reichsuntertanen grundsätzlich offenstehen, allerdings nicht notwendigerweise im Rahmen einer förmlichen Appellation. In den Quellen wird ein weiter Appellationsbegriff verwendet, der auch eine appellatio extrajudicialis einschließt. Es findet sich regelmäßig auch der Terminus recursus/Rekurs.

Anders als bei informellen Rekursen stand einer förmlichen Appellation an den Kaiser das eingebürgerte italienische Appellationsverfahren entgegen, das keinen klaren Instanzenzug vorsah und sich insofern deutlich von der deutschen Praxis unterschied. Dazu kamen einige praktische Probleme, insbesondere der Umstand, dass der Aufwand eines Reichshofratsprozesses oder auch nur einer Vergleichsverhandlung vor dem Plenipotentiar kaum im Verhältnis zu einem oft nur geringen Streitwert stand und die finanziellen Möglichkeiten vieler Untertanen weit überstieg. Vor diesem Hintergrund wäre zu erwarten, dass förmliche Appellationsverfahren aus Italien für den Reichshofrat keine große Rolle gespielt haben dürften.

Einer pragmatischen Lösung des Problems stand der grundsätzliche Widerwille des Reichs-

davon, dass es Joseph II. „teilweise gelungen“ sei, die Widerstände der Malaspina gegen die Zulassung von Klagen ihrer Untertanen an der Plenipotenz zu überwinden. 
hofrats entgegen, dem Plenipotentiar in Justizangelegenheiten einen Status zuzugestehen, der über den eines kaiserlichen Generalkommissars hinausging. Insbesondere widersetzte sich der Reichshofrat energisch allen Ansätzen, die Plenipotenz in ein ordentliches Gericht zu transformieren. Deutlich werden seine Bedenken erkennbar, sich durch eine Neuregelung des Appellationsverfahrens auf einen Konflikt mit den größeren Reichsvasallen einzulassen. Die Belange der minderbemittelten Vasallen und der Untertanen wurden diesem Interesse untergeordnet. Im Grunde kreisten die Diskussionen zwischen Plenipotentiaren, italienischen Reichsfiskalen und den Wiener Reichsbehörden jahrzehntelang um dieselben Probleme. Dabei lässt sich die Tendenz erkennen, dass dem Reichshofrat neben der Wahrung der eigenen Kompetenzen und Interessen auf einer eher abstrakten Ebene v.a. die Wahrung des kaiserlichen höchstrichterlichen Ansehens am Herzen lag, während die Amtsträger vor Ort praxisnäher und insbesondere mit dem Anliegen eines effektiven Untertanenschutzes argumentierten.

Zwar wurden die Kompetenzen von Plenipotentiar und Reichsfiskal in Italien allmählich deutlicher umschrieben und partiell ausgeweitet. Kennzeichnend für sie war jedoch im Justizbereich der Status einer Untersuchungskommission mit begrenzter Mediationskompetenz. Jedenfalls bis in die 1780er Jahre hatten die wiederholten Vorstöße von Plenipotentiaren und italienischen Fiskalen wenig daran ändern können. Inwieweit die Vermittlung der Plenipotenz in Appellationsfällen (und anderen Rechtsstreitigkeiten) von den Parteien in Anspruch genommen wurde, ob und in welchem Umfang direkt nach Wien appelliert wurde oder sich die ältere Appellationspraxis behauptete, bliebe jedoch zu erforschen.

Überhaupt müsste es sehr viel mehr Fallstudien geben, um das Bild der Appellationen aus Italien und allgemein der Reichsgerichtsbarkeit in Italien klarer zu konturieren. Neben qualitativen
Untersuchungen wären aber auch quantitative Erhebungen sinnvoll, um besser beurteilen zu können, wie häufig und in welchen Fällen der Reichshofrat aktiv wurde. $\mathrm{Zu}$ vermuten wäre, dass den Extrajudizialappellationen eine besondere Bedeutung zukam.

Wesentlich genauer müsste insbesondere die Rolle der Plenipotenz erforscht werden. Hierzu wäre es dringend erforderlich, die Überlieferung im Archivio di Stato di Milano und möglichst auch die Privatarchive der Plenipotentiare in die Untersuchung mit einzubeziehen. Neben dem spannungsreichen Verhältnis zum Reichshofrat könnte so die Bedeutung der Plenipotenz als Vermittlungs- und Schutzinstanz für Untertanen und mindermächtige Vasallen deutlicher ins Licht treten. Außerordentlicher Bedarf besteht an Forschungen zum 16. und 17. Jahrhundert, also für die Zeit vor der Plenipotenz. Auch hier müssten neben den Beständen des Wiener Reichshofratsarchivs auch italienische Quellen ausgewertet werden, beispielsweise die Überlieferungen der Generalkommissare der ersten Hälfte des 17. Jahrhunderts.

Solche und weitere Untersuchungen könnten einen Beitrag zum übergeordneten Forschungsproblem leisten, welche Rolle Italien für das Reich und welche Rolle das Reich für Italien in der Frühen Neuzeit spielte. Vielleicht gelangt dann auch die moderne historische und rechtshistorische Forschung zu klareren Einschätzungen, als sie den Reichspublizisten des späten 18. Jahrhunderts möglich waren.

\section{Korrespondenz:}

Prof. Dr. Matthias Schnettger Johannes Gutenberg Universität Mainz Fachbereich 07 Geschichts- und Kulturwissenschaften Historisches Seminar - Neuere Geschichte Jakob-Welder-Weg 18, 55128 Mainz, Deutschland Schnettger@uni-mainz.de 


\section{Abkürzungen:}

Siehe das allgemeine Abkürzungsverzeichnis: http://www.rechtsgeschichte.at/files/abk.pdf

\section{Literatur:}

Karl Otmar von ARETIN, Das Alte Reich 1648-1806, Bde. 1-4 (Stuttgart 1993-2000).

DERS., Reichsitalien von Karl V. bis zum Ende des Alten Reiches. Die Lehensordnungen in Italien und ihre Auswirkungen auf die europäische Politik, in: DERS., Das Reich. Friedensgarantie und europäisches Gleichgewicht 1648-1806 (Stuttgart 1986) 76-163.

Tommaso di CARPEgna FALCONIERI, Die Reichslehen der Grafen und Fürsten von Carpegna (13. bis 19. Jahrhundert), in: zeitenblicke 6 (2007) Nr. 1 [http://www.zeitenblicke.de/2007/1/carpegna/ index_html], URN: urn:nbn:de:0009-9-8042 (2007, abgerufen am 13. 04. 2012).

Guido Del PINO, Un problema burocratico: La Plenipotenza per i feudi imperiali in Italia e il suo archivio tra XVII e XVIII secolo, in: Rassegna degli Archivi di Stato 54 (1994) 551-583.

Johann August Ritter von EISENHART, Art. Seidensticker, Johann Anton Ludwig, in: ADB, Bd. 33 (Leipzig 1891) 630.

Gesine Göschel, Das ,Bellum Diplomaticum' um Comacchio zu Beginn des 18. Jahrhunderts (jur. Diss., Univ. Frankfurt am Main 1973).

Gernot Peter OBERSTEINER, Das Reichshoffiskalat 1696 bis 1806 . Bausteine zu seiner Geschichte aus Wiener Archiven (Staatsprüfungsarbeit, Institut für Österreichische Geschichtsforschung Wien 1992), gekürzt auch in Anette BAUMANN u.a. (Hgg.), Reichspersonal. Funktionsträger für Kaiser und Reich (= Quellen und Forschungen zur höchsten Gerichtsbarkeit im Alten Reich 46, Köln-WeimarWien 2003) 89-164.

Ugo Petronio, Il Senato di Milano. Istituzioni giuridiche ed esercizio del potere nel Ducato i Milano da Carlo V a Giuseppe II, Bd. 1 (= Ius nostrum 17,1, Milano 1972).
Salvatore PUGLIESE, Le prime strette dell'Austria in Italia (Milano 1932, 2. Aufl. unter dem Titel: Il Sacro Romano Impero in Italia ebd. 1935).

Gerhard RILL, Reichsvikar und Kommissar. Zur Geschichte der Verwaltung Reichitaliens im Spätmittelalter und in der frühen Neuzeit, in: Annali della Fondazione italiana per la Storia amministrativa 2 (1965) 173-198.

Matthias SCHNETTGER, "Abschied von Germania“. Italienische Perspektiven auf das Ende des Alten Reiches, in: Christine ROLL, DERS. (Hgg.), Epochenjahr 1806? Das Ende des Alten Reichs in zeitgenössischen Perspektiven und Deutungen (Veröffentlichungen des Instituts für Europäische Geschichte Mainz, Beiheft 76, Abteilung für Universalgeschichte, Mainz 2008) 41-59.

DERS., Das Alte Reich und Italien in der Frühen Neuzeit. Ein institutionengeschichtlicher Überblick, in: Quellen und Forschungen aus italienischen Archiven und Bibliotheken 79 (1999) 344-420.

DERS., Kooperation und Konflikt. Der Reichshofrat und die kaiserliche Plenipotenz in Italien, in: Anja AMEND u.a. (Hgg.), Gerichtslandschaft Altes Reich. Höchste Gerichtsbarkeit und territoriale Rechtsprechung (= Quellen und Forschungen zur höchsten Gerichtsbarkeit im Alten Reich 52, KölnWeimar-Wien 2007) 127-149.

DERS., „Principe sovrano" oder „civitas imperialis“? Die Republik Genua und das Alte Reich in der Frühen Neuzeit (1556-1797) (= Veröffentlichungen des Instituts für Europäische Geschichte 209, Abteilung für Universalgeschichte; Beiträge zur Sozial- und Verfassungsgeschichte des Alten Reiches 17, Mainz 2006).

Johann Anton Ludwig SEIDENSTICKER, Beyträge zum Reichsstaatsrechte Welscher Nation, Bd.1 [mehr nicht erschienen] (Göttingen 1795).

Angelo TAMBORRA, La pace di Aquisgrana del 1748 e la politica della Santa Sede, in: Archivio Storico Italiano 117 (1959) 522-540.

Wilhelm WEGENER, Die staatsrechtliche Stellung der italienischen Reichsteile am Ende des alten Reiches im Spiegel der späten Reichspublizistik, in: Norbert ACHTERBERG, Werner KRAWIETZ, Dieter WYDUCKEL (Hgg.), Recht und Staat im sozialen Wandel. Festschrift für Hans Ulrich Scupin zum 80. Geburtstag (Berlin 1983) 195-208. 


\section{Zusammenfassung}

Appellationen von italienischen Vasallen und Untertanen des Heiligen Römischen Reichs sind im Kontext der kaiserlichen Jurisdiktion im sog. Reichsitalien zu sehen, die mit außerordentlich schwierigen Rahmenbedingungen zu kämpfen hatte. Während der Reichshofrat und die Plenipotenz in Italien dazu dienen sollten, die kaiserliche Jurisdiktionsgewalt über alle Reichslehen zu betonen, stieß die Durchsetzung dieser Ansprüche auf Schwierigkeiten: Insbesondere die größeren Vasallen (aber nicht nur sie) versuchten, den Zugang ihrer Untertanen zu den Reichsgerichten abzuschneiden. Darüber hinaus bemühte sich der Reichshofrat, die Entwicklung der Plenipotenz in eine ordentliche, reguläre Instanz zu verhindern und ihren Status als untergeordnete, hilfsweise tätige Untersuchungskommission zu erhalten, während eine Appellation nach Wien für die meisten Untertanen wegen der hohen Kosten jenseits ihrer Möglichkeiten war. Schließlich sah die eingeführte Praxis in Italien für Appellationen keine festen Stationen vor, sondern ermöglichte es den Vasallen, von Fall zu Fall gemäß ihren Interessen Appellationsgerichte einzusetzen. Angesichts dieses Herkommens scheinen reguläre Appellationen aus Italien am Reichshofrat eine geringe Rolle gespielt zu haben, während Extrajudizialappellationen möglicherweise häufiger vorkamen. Es bleibt allerdings noch viel zu tun, um die Bedeutung reichsitalienischer Appellationen und, allgemeiner, der kaiserlichen Jurisdiktion in Reichsitalien angemessen bewerten zu können - nicht zuletzt hinsichtlich der gerichtlichen und vermittelnden Tätigkeit der Plenipotenz.

\section{Summary}

Appeals by the Italian vassals and subjects of the Holy Roman Empire have to be considered within the wider context of imperial jurisdiction in the so-called "Reichsitalien" which had to cope with an especially difficult general situation. While the Imperial Aulic Council ("Reichshofrat") and the "Plenipotenz" in Italy were used to emphasize the Emperor's supreme jurisdiction over all the imperial fiefs, it was quite difficult for them to sustain their claims, particularly because the major vassals (but not only they) sought to block their subjects' access to Imperial courts. Furthermore, the Aulic Council was anxious to prevent the evolution of the Plenipotenz into a regular, independent instance and sought to keep it in a subordinate status as an auxiliary investigating commission. In any event, for most subjects an expensive appeal to Vienna was beyond their means. Finally the established Italian practice did not include precise stages of appeal but rather allowed vassals to nominate appellate courts from case to case, according to their interests. In light of these observations, regular, jurisdictional appeals by Italian subjects seem to have played a minor role within the jurisdiction of the Aulic Council, although extra-jurisdictional appeals may have been more common. But there is still much work to do to evaluate the importance of the Italian appeals and, more generally, of the imperial jurisdiction in Italy, not least with regard to the jurisdictional and mediating practices of the Plenipotenz. 